\title{
Interferometric Observations of $\eta$ Carinae - the VLTI Takes Its First Glimpse at the Central Source
}

\author{
P. Kervella, M. Schöller \\ European Southern Observatory, Casilla 19001, Santiago 19, Chile \\ R. van Boekel, E. di Folco, S. Correia, A. Glindemann, F. Paresce, \\ A. Richichi, M. Tarenghi \\ European Southern Observatory, Karl-Schwarzschild-Str. 2, \\ 85748 Garching, Germany
}

\author{
M. Wittkowski \\ European Southern Observatory, Casilla 19001, Santiago 19, Chile
}

\begin{abstract}
We describe the first long-baseline interferometric measurement ever made of the luminous blue variable $\eta$ Carinae. $\eta$ Car was observed with the Very Large Telescope Interferometer (VLTI) with the $8.2 \mathrm{~m}$ Unit Telescopes as well as with the $40 \mathrm{~cm}$ test siderostats on three different baselines. We will detail the measurements and data analysis and discuss briefly the implications of the derived visibility on the morphology of the central source. Furthermore we will discuss the future possibilities of interferometric observations and will show that $\eta$ Car is one of the prime candidates for imaging with the VLTI.
\end{abstract}

\section{1. $\eta$ Carinae}

$\eta$ Carinae is the most massive, most luminous known star in our region of the Milky Way. In the last nearly two hundred years $\eta$ Car has lived through a turbulent history. Its biggest eruption in the 1840 s has led to the surrounding bipolar homunculus nebula. However, the cause of this outburst is still not known. The study of $\eta$ Car raises important questions about how the very most massive stars may end their lives. Some progress has been made in understanding the morphology of the nebula, but its formation is still under controversy (Davidson et al. 2001). The central source was studied by high angular resolution techniques, finding a complex structure, e.g. equatorial blobs, which brightness did not fit into the general picture (Weigelt \& Ebersberger 1986; Falcke et al. 1996). The detection of a 5.5-year period in spectroscopy and near-infrared photometry (Damineli 1996), hinting at shell events, has led to new disputes. In the last years different observations and interpretations range from a binary system to variations coming from the ejecta around the source (Damineli et al. 2000; Davidson et al. 2000). The detection of a well defined torus at 10 micron 
(Morris et al. 1999; Hony et al. 2001) has been questioned by observations with higher spatial resolution (Smith et al. 2002).

\section{Observing $\eta$ Carinae with VLTI and VINCI}

The Very Large Telescope Interferometer (Glindemann et al. 2000), located on Cerro Paranal (Chile) is the largest astronomical instrument working at optical wavelengths in the world. It comprises four Unit Telescopes (UT, $8.2 \mathrm{~m}$ diameter) and three movable Auxiliary Telescopes (AT, $1.8 \mathrm{~m}$ diameter). The ATs can be positioned on thirty different stations, giving a high flexibility in the array configuration. Through the commissioning phase two $40 \mathrm{~cm}$ siderostats are used on a subset of the AT stations. The delay line tunnel can house eight delay lines, three of which are currently operational. Fig. 1 shows the layout of the VLTI.

VLTI had first fringes with the two test siderostats on March 17, 2001 on a $16 \mathrm{~m}$ baseline, and with the two UTs UT1 (named Antu) and UT3 (Melipal), spanning a $102 \mathrm{~m}$ baseline, on October 29,2001. On both baselines as well as with the siderostats on a $66 \mathrm{~m}$ baseline (since early 2002), more than 150 different objects were observed with the VLT INterferometric Commissioning Instrument (VINCI). VINCI (Kervella et al. 2000) is a two telescope fiber beam combiner, which operates in the $\mathrm{K}$ band at an effective wavelength of $2.13 \mu \mathrm{m}$.

$\eta$ Car was observed with VLTI and VINCI on all three different baselines mentioned above during four nights. The test siderostats were positioned on the stations $\mathrm{E} 0$ and $\mathrm{G} 0$, and $\mathrm{E} 0$ and $\mathrm{G} 1$, which give baselines of $16 \mathrm{~m}$ and $66 \mathrm{~m}$, respectively. A detailed observation log is given in Table 1.

\begin{tabular}{ccc} 
JD & Reduced scans & Telescopes \\
\hline & & \\
2452216.864 & 50 & UT1/UT3 \\
2452216.867 & 44 & UT1/UT3 \\
2452302.880 & 44 & E0/G0 \\
2452302.885 & 32 & E0/G0 \\
2452304.824 & 159 & UT1/UT3 \\
2452322.581 & 12 & E0/G1 \\
2452322.621 & 9 & E0/G1 \\
2452322.625 & 5 & E0/G1 \\
2452322.865 & 7 & E0/G1 \\
2452322.899 & 3 & E0/G1
\end{tabular}

Table 1. $\quad \eta$ Carinae data sets taken with the VLTI and VINCI between November 2001 and February 2002.

Given the geometrical configuration of the test siderostats, the observation of objects located in the south and relatively low above the horizon is difficult. This effect is due to vignetting of the incoming beams by the siderostat structures, and explains partly the low number of reduced scans of the siderostat measurements compared to the UT measurements. Especially the data observed on the E0/G1 baseline suffer from this restriction together with the lower visi- 


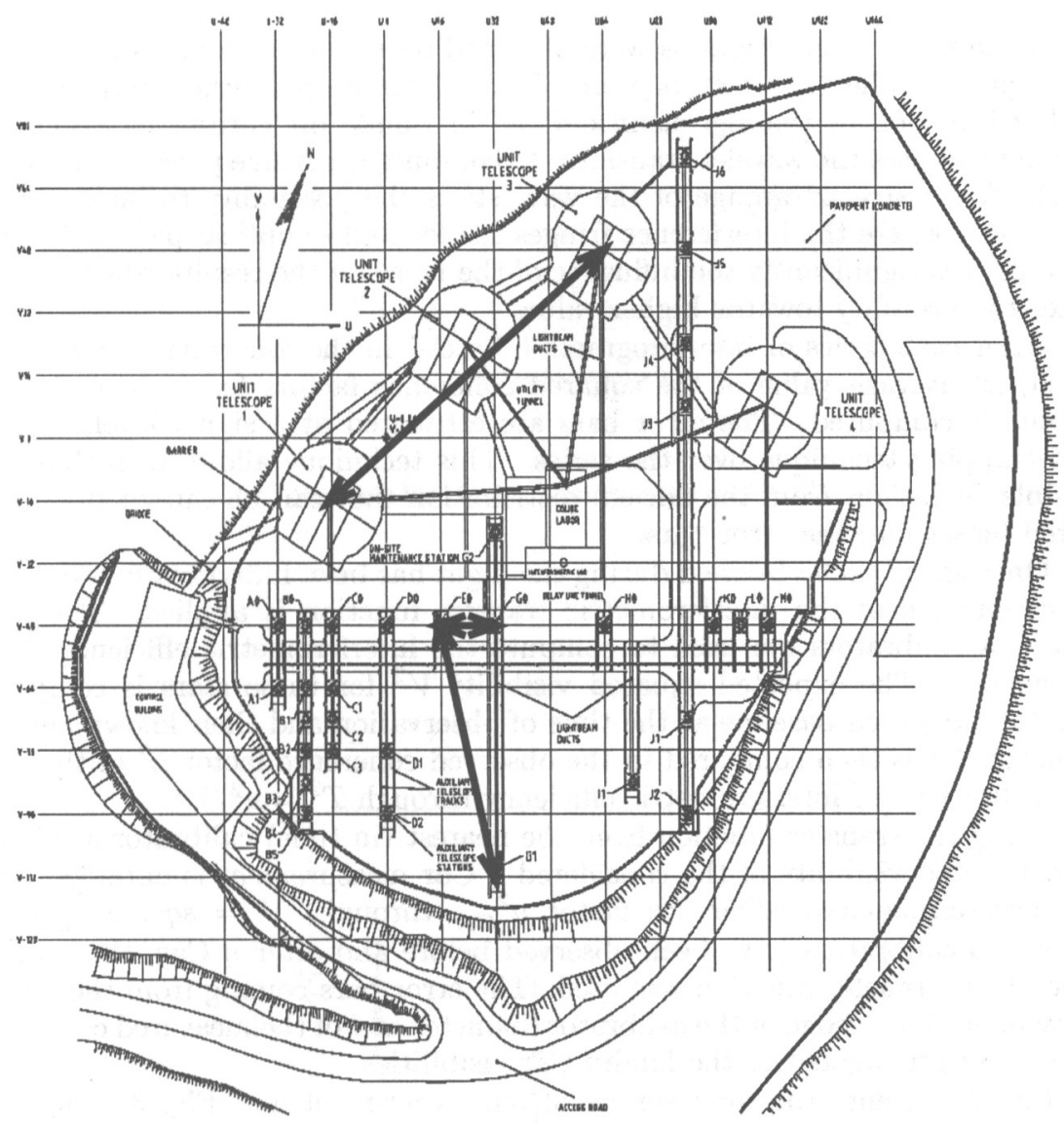

Figure 1. Overview of the VLTI complex. Displayed are the four UTs and the $30 \mathrm{AT}$ stations. The AT stations are connected by rail tracks on which the ATs can be moved. The delay line tunnel can be seen in the center of the drawing crossing the whole mountain top eastwest, with the beam combination laboratory just north of it. The thick lines with arrows denote the baselines used within our observations, spanning $16 \mathrm{~m}, 66 \mathrm{~m}$, and $102 \mathrm{~m}$. 
bility on this longer baseline, and are thus less reliable than the data taken on other baselines.

\section{Data Reduction}

Several series of interferograms were obtained on $\eta$ Carinae and its calibrators. The squared coherence factors were obtained for each interferogram using a modified version of the algorithm defined in Coudé du Foresto et al. (1997). This version uses the wavelet transform to compute the fringe power (Ségransan, 2001). A decisive advantage of the wavelets is the possibility to integrate the power only where the interference fringes are located in optical path difference. This reduces significantly the influence of the noise on the results which usually biases the visibility towards higher values.

After each series of interferograms ("batch" in the following) has been reduced, an average value of the squared coherence factors for each of the two outputs is computed. The error bars are estimated at 3 sigma level, using a bootstrapping technique over the series. This technique allows to include the possible deviations from the normal distribution that can be caused by uncalibrated biases into the error bars.

Once each batch obtained during the night has been individually processed, the calibration of the interferometric transfer function is applied. The stars chosen as calibrators are used to compute the interferometric efficiency $T^{2}$ of the system. The expected squared visibility $V^{2}$ for these stars is computed from the projected baseline at the time of observation and their known angular diameter. $V^{2}$ is then compared to the observed coherence factor $\mu^{2}$ of the star, giving directly the interferometric efficiency through $T^{2}=\mu^{2} / V^{2}$.

Using the transfer function from the nearest (in time) calibrator available, the calibrated visibility of the considered $\eta$ Car measurement is obtained from the observed squared coherence factor $\mu_{\eta C a r}^{2}$ through: $V=\operatorname{sqrt}\left(\mu_{\eta C a r}^{2} / T^{2}\right)$. When two calibrators have been observed before and after $\eta$ Car, the average value of the transfer function was used. The error bars coming from the uncertainty in our knowledge of the calibrator diameter and of the measured coherence factor were propagated to the final $\eta$ Car visibilities.

Fig. 2 presents the $u v$-coverage of our experiment and Fig. 3 shows the visibilities measured on the different baselines.

\section{Interpretation}

The light distribution of $\eta$ Carinae seems to be built up out of (at least) two components: a compact central source with emission on a scale of a few milliarcseconds (mas), i.e. about $10 \mathrm{AU}$, and a more extended component which is at least largely resolved on the shortest baseline, where spatial scales of about 30 mas are probed. A one component model can not explain the relatively low visibility at the short baseline and the relatively high visibilities at longer baselines. Within this interpretation, the compact component is emission that directly emerges from the central star (or it's wind). The large component is likely reprocessed radiation: thermal emission from dust, which is expected consider- 


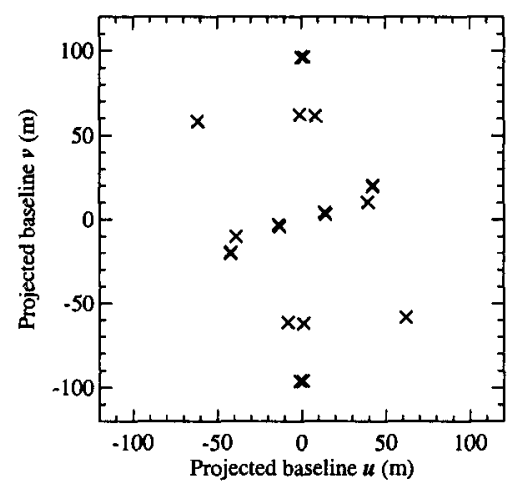

Figure 2. $u v$-coverage for the observations of $\eta$ Carinae with the VLTI and VINCI.

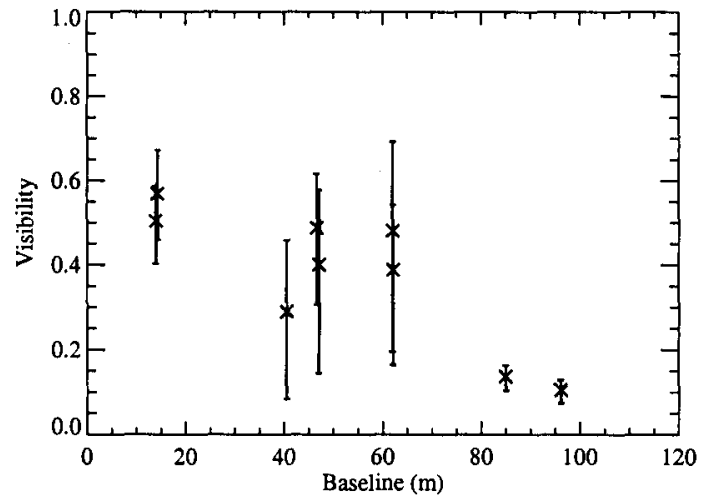

Figure 3. Visibilities determined with the VLTI and VINCI for $\eta$ Carinae in the $\mathrm{K}$ band. Note the small error bars on the longest baselines with the Unit Telescopes, while especially the $66 \mathrm{~m}$ baseline with the test siderostats shows very large errors (see text). 
ing the very red spectral energy distribution (SED) of the central arcsecond of $\eta$ Car (Rigaut \& Gehring 1995). More observations on different points in the $u v$ plane and more detailed analysis and modelling are required to give a definitive answer.

Note that a model involving a detectable secondary is very unlikely, since previous studies have shown that at NIR wavelengths the spectrum of $\eta \mathrm{Car}$ is fully dominated by the primary.

Interpretation of the results taken so far on $\eta$ Car suffers for various aspects: The effective field of view on sky fed into the fibers of the VINCI instrument depends on the Airy disk of the individual telescope and is thus different for the siderostats and the Unit Telescopes by a factor of 20 . When modelling the visibilities one has to take this fact into account. Due to the design of the test siderostats, which are optimized to look north, $\eta$ Car is partially vignetted. This reduces the flux, which is already close to the limit observable with the siderostats in early 2002. Especially on the longer baseline with reduced coherence this leads to less sensitivity, and hence less scans above the rejection threshold. In a recent paper, Guyon (2002) showed that interferometric observations of extended sources with a fiber fed beam combiner suffer from variations of the atmospheric seeing, especially on large individual telescopes which partially resolve the source. Finally, within our analysis we are actually assuming a rotational symmetry of the object, which very likely can not hold given the bipolar shape on large scales and the different asymmetrical structures found on small scales. Even with a more detailed model one is bound to point symmetrical objects before closure phases (Jennison 1958) become available with the AMBER instrument (Petrov et al. 2000).

\section{Conclusion and outlook}

Using the VLTI together with VINCI on several different baselines allows us for the first time to probe the central source of $\eta$ Carinae with unprecedented resolution. Although interferometric observations of extended objects are difficult and the position of $\eta$ Car in the sky is not optimal for VLTI observations with siderostats, we were able to measure the visibility function on several places of the $u v$-plane for three different baselines. In fact, VLTI is the only large interferometer that can look at $\eta$ Car.

The central object is apparently well resolved with the UT's $102 \mathrm{~m}$ baseline. We plan to confront this result with models in the future. More intermediate baseline lengths and a good azimuthal coverage will allow us to detect any asymmetry in the core of the source, at milli-arcsecond level.

As the next shell episode of $\eta$ Car is expected in June 2003, we will collect more data to be able to trace the visibility values over $\eta$ Car's evolution cycle.

These first results obtained with VINCI and the VLTI on only three different baselines give us confidence that we can actually probe the central source using the interferometric technique. In the near future we will obtain visibility measurements on several other baselines, to define better the flux distribution of $\eta$ Car. Since $\eta$ Car has a complex light distribution, new approaches to the analysis of the visibility data have to be made. At the resolution level probed by the VLTI baselines (up to $202 \mathrm{~m}$ ), many objects currently appearing to us 
as point-like will suddenly show complex and changing structures. VLTI will give us the possibility to observe for example heavily obscured stars, interacting binaries or star spots. The AMBER instrument, expected to be installed on Paranal in the first half of 2003 , will be able to measure closure phases. Together with medium resolution spectral dispersion of about 10,000 and access to $\mathrm{J}$ and $\mathrm{H}$ band, this will improve tremendously the capabilities of VLTI and allow us to reconstruct the image of $\eta$ Car with previously unmatched detail.

\section{References}

Coudé du Foresto, V., Ridgway, S., \& Mariotti, J.-M. 1997, A\&A, 121, 379

Damineli, A. 1996, ApJ, 460, L49

Damineli, A., Kaufer, A., Wolf, B., Stahl, O., Lopes, D. F., \& de Araújo, F. X. 2000, ApJ, 528, L101

Davidson, K., Ishibashi, K., Gull, T. R., Humphreys, R. M., \& Smith, N. 2000, ApJ, 530, L107

Davidson, K., Smith, N., Gull, T. R., Ishibashi, K., \& Hillier, D. J. 2001, AJ, 121,1569

Delplancke, F., Leveque, S. A., Kervella, P., Glindemann, A., \& D'Arcio, L. 2000, SPIE, 4006, 365

Falcke, H., Davidson, K., Hofmann, K.-H, \& Weigelt, G. 1996, A\&A, 306, L17

Glindemann, A., et al. 2000, SPIE, 4006, 2

Guyon, O. 2002, A\&A, 387, 366

Hony, S., et al. 2001, A\&A, 377, L1

Jennison, R. C. 1958, MNRAS, 118, 276

Kervella, P., Coudé du Foresto, V., Glindemann, A., \& Hofmann, R. 2000, SPIE, 4006,31

Morris, P. W., et al. 1999, Nature, 402, 502

Petrov, R. G., et al. 2000, SPIE, 4006, 68

Rigaut, F., \& Gehring, G. 1995, Rev. Mex. Astron. Astrof. Ser. Conf., 2, 27

Ségransan, D. 2001, Ph.D. thesis, Grenoble

Smith, N., Gehrz, R. D., Hinz, P. M., Hoffmann, W. F., Mamajek, E. E., Meyer, M. R., \& Hora, J. L. 2002, ApJ, 567, L77

Weigelt, G., \& Ebersberger, J. 1986, A\&A, 163, L5 